\title{
La rete mediterranea della devozione. Le teresiane della provincia di Napoli
}

\section{The Mediterranean network of devotion. Teresian nuns from the Neapoletan interland}

\author{
VITTORIA FIORELLI \\ vittoria.fiorelli@unisob.na.it \\ Università degli studi Suor Orsola Benincasa di Napoli
}

\begin{abstract}
Riassunto: Filo conduttore del saggio è la vita di madre Serafina di Dio, carismatica caprese fondatrice, nel secolo XVII, di sette congregazioni femminili ispirate alla spiritualità teresiana. L'affiliazione alla santa di Avila e l'adesione alla matrice della sua riforma hanno consentito alle nuove comunità devote nate da un'iniziativa periferica di collocarsi nell'alveo della Chiesa nella prospettiva istituzionale della trasformazione in monasteri di clausura. Due i focus della ricerca. Da un lato ci si è soffermati sull'omogeneità organizzativa e spirituale dei conservatori garantita da reti devozionali saldamente dipendenti dal carisma della fondatrice secondo un meccanismo nel quale resta assai difficile determinare i confini tra una vita modellizzata dalla santità e il progetto di santificazione della propria vita. Dall'altro si è evidenziata l'importanza della tradizione religiosa e devozionale nel processo di costante contaminazione dell'identità culturale delle due principali penisole del Mediterraneo mai trasformata in fusione, nonostante gli intensi rapporti e la subalternità politica di Napoli a Madrid.
\end{abstract}

Parole chiave: reti devozionali e religiose, spiritualità femminile, Mediterraneo.

Abstract: The underlying theme of the essay is the life of Mother Serafina di Dio from Capri, the charismatic founder, in the seventeenth century, of seven women's congregations inspired by the Teresian spirituality. The affiliation to the saint of Avila and the connection to the matrix of her reform allowed these peripheral and devoted communities to position themselves within the fold of the Church, from the perspective of institutional transformation in cloistered monasteries. The first research focus is about the uniformity of organization and spirituality within the retreats guaranteed by devotional networks firmly dependent on the charisma of the founder. A mechanism in which is very difficult to determine the boundaries between a life modeled on the holiness and a project of sanctification of her own life. The other one, is about the importance of religious and devotional traditions in the process of constant contamination of the cultural identity of the two main peninsulas in Mediterranean, never turned into fusion, despite the penetrating contacts and the political subordination of Naples in Madrid.

Keywords: religious and devotional networks, women's spirituality, Mediterranean

DATA PRESENTACIÓ: 09/12/2015 ACCEPTACIÓ: 15/12/2015 ·PUBLICACIÓ: 26/12/2015

SCRIPTA, Revista internacional de literatura i cultura medieval i moderna, núm. 6 / desembre 2015 / pp. 155 - 165 ISSN: 2340-4841 · doi:10.7203/SCRIPTA.6.7828 
La mattina del 29 maggio 1661 otto donne coronate di spine uscivano in processione dalla chiesa di Santo Stefano a Capri per ritirarsi a vivere in una casa non lontana, messa a loro disposizione da uno dei notabili della piccola comunità. Le guidava una non giovanissima bizzoca, Prudenza Pisa, e le accompagnavano due illustri prelati, Orazio D'Amato, inviato sull'isola in qualità di vicario apostolico per sostituire il vescovo Paolo Pellegrino, e Ottavio Pisa, canonico della cattedrale di Napoli e parente della carismatica caprese ${ }^{1}$.

Registrato con grande risalto dalle fonti coeve, l'episodio segnava l'atto di nascita di un «conservatorio con educandato» dal quale si sarebbe sviluppata la storia delle «teresiane» di madre Serafina di Dio. Un evento certamente importante nella vita isolana, destinato a superare la proiezione della vicenda religiosa periferica per trasformarsi nel percorso istituzionale di una tradizione spirituale moderna, carica di significati devozionali, politici e culturali.

Terra del mito capace di esercitare un incanto magnetico sui viaggiatori che sono approdati sulle sue coste, Capri, che pur vanta una ricca e variegata bibliografia, conta rari contributi dedicati alla sua storia. Un vuoto storiografico riconducibile alla marginalità che, conclusa la parabola romana delle residenze imperiali, l'isola ha subito durante i secoli del medioevo e dell'età moderna, nonostante la posizione centrale nel golfo di Napoli e lungo le grandi rotte del Mediterraneo, soprattutto a causa della scarsezza delle sue risorse. Ridotta a poco più di un faro per i naviganti, a più riprese destinata a luogo di pena e di confino, la povertà e la litigiosità delle due comunità che vivevano sul territorio la aveva portata a dipendere completamente dalla capitale distante solo poche ore di feluca ${ }^{2}$.

Diocesi dalla fine del X secolo, Capri era dominata dalla potente Certosa di S. Giacomo dotata, fin dal XIV secolo, di ampi privilegi e di ingenti risorse economiche. Oltre ai certosini, si erano stabilite sull'isola piccole comunità regolari che incidevano pochissimo sulla vita devozionale e sociale dei centri urbani, mentre non esisteva, ancora in pieno Seicento, alcuna struttura di accoglienza per le donne che volessero seguire una vita ritirata ${ }^{3}$.

\author{
1 Abbreviazioni: \\ ACDF: Archivio della Congregazione per la Dottrina della Fede, Città del Vaticano \\ APC: Archivio Parrocchiale di Capri \\ ASNa: Archivio di Stato di Napoli \\ BCIC: Biblioteca del Centro Caprense Ignazio Cerio, Capri \\ CADC: Centro Archivistico Documentale Isola di Capri
}

Sulla vicenda delle teresiane di Capri e su madre Serafina di Dio mi sia consentito rinviare ai lavori di chi scrive, soprattutto V. Fiorelli, Una esperienza religiosa periferica. I monasteri di madre serafina di Dio da Capri alla terraferma, Napoli 2003 al quale si può fare riferimento per una più ampia bibliografia e per il riferimento puntuale alle fonti.

2 C. Sandomenico, Leggere Capri. Bibliografia dellisola di Capri, Capri 1993 offre una ricca rassegna della letteratura su Capri. Per una sintetica lettura storiografica si veda G. Galasso, Capri insula e dintorni, Capri 2004.

3 Della diocesi di Capri si ha notizia dal 987 e fu soppressa nel 1818 quando fu accorpata a quella di Sorrento. Su questo B. Capasso, Memorie storiche della Chiesa sorrentina, Napoli 1854. La certosa, beneficata dal diploma di Giovanna I nel 1371 e dalla bolla di Gregorio XI nel 1375, dimostrava, ancora nel 1563, la preminenza non solo economica del 
In questo contesto, funestato da un violento contenzioso giurisdizionale tra il vescovo Paolo Pellegrino e i rappresentanti dell'università, prese forma, all'interno del tessuto sociale isolano, il progetto di fondare un conservatorio che accogliesse giovanette devote e desse loro un'educazione religiosa tenendole al riparo dai pericoli del secolo. La storia della sua realizzazione e la capacità di proiettare una gemmazione di iniziative oltre i confini della piccola isola offre agli studiosi l'opportunità di seguire alcuni percorsi storiografici di notevole interesse e di intrecciare l'evoluzione della comunità religiosa di Capri con il più ampio sviluppo della cultura devota e spirituale dei territori immediatamente contigui alla capitale, eppure segnati da una marcata dimensione periferica.

Il primo ideatore di questo programma era stato il sacerdote Marcello Strina, arcidiacono del capitolo cattedrale, confessore e parroco a Capri dal 1653 fino alla sua morte, durante la violenta epidemia di peste che, tre anni più tardi, falcidiò la popolazione dell'isola, così come quella dell'intera regione. Nella sua realizzazione sebbene secondo un percorso non perfettamente in sintonia con quello inizialmente ipotizzato, pesò non poco il contributo di Ottavio Pisa al quale lo legavano reti familiari ${ }^{4}$.

I due ecclesiastici condividevano il legame di parentela con Prudenza, figlia di Colantonio, cugino di don Ottavio, nata a Napoli il 24 ottobre 1621 da un mercante al secondo matrimonio che, in seguito a un processo per fallimento, aveva trasferito la famiglia a Capri dove la moglie, Giustina Strina, possedeva tanto da vivere con decoro. Appartenente a una delle famiglie più rilevanti dell'isola, affidata alle cure dello zio parroco che le fece da guida spirituale e confessore, la giovane ricevette un'educazione di buon livello che le avrebbe consentito un inusuale e costante uso della scrittura della quale si sarebbe servita per costruire il suo ruolo di monaca fondatrice.

L'influenza, in momenti diversi, dei due religiosi fece in modo che la serva di Dio coltivasse, accanto al modello di spiritualità carismatica e penitenziale diffuso tra le donne che si sentivano chiamate a un percorso privilegiato di abbandono devoto, l'aspirazione a guidare una comunità regolare femminile nell'alveo istituzionale della Chiesa trionfante ${ }^{5}$.

suo ruolo isolano con l'autorizzazione vicereale a costruire una torre di difesa. Sempre utile il contributo di storiografia municipale E. Cerio, Capri nel Seicento. Documenti e note, Napoli 1934, ma si ceda anche G. Ruocco, Capri nei suoi documenti archivistici (sec. XV-XVI-XVII), Napoli 1952; Fiorelli, Una esperienza religiosa periferica cit., pp. 7-41. Per evitare continui rinvii, sono state omesse le continue citazioni di questo volume che resta il riferimento necessario per approfondire le vicende trattate di seguito.

4 Nel registro dei morti della chiesa di Santo Stefano di Capri è indicata una sequenza apposita titolata Tempore pestis che inizia il 3 giugno del 1656. APC, Libro dei morti. 1638-1692, ff. 22v.-26. Da questo elenco risulta il decesso di cinque ecclesiastici: Giovanni Andrea de Leo, Carlo Porrato, Andrea Ferrace, Pietro Cola Arcuccio e Marcello Strina. Ottavio Pisa ha lasciato testimonianza del rapporto con Prudenza nel manoscritto Libro del Monasterio delle Monache del SS. Salvatore della Città di Capri scritto dal Canonico D. Ottavio Pisa Penitenziere maggiore della Chiesa Cattedrale di Napoli, e Consultore del S. Officio nell'Anno 1668, una copia del quale è in CADC 91.A.4.

5 Ancora attuale I. Poutrin, Le voile et la plume. Autobiographie et sainteté féminine dans l'Espagne moderne, Madrid 1995. Per limitarci al contesto dell'Italia meridionale si veda M. Campanelli, «Una virtù soda, maschia e robusta» Il monachesimo femminile nel Settecento napoletano e V. Fiorelli, Cupio dissolvi. Destini di donne tra profetismo e ascesi monastica, entrambi in G. 
L'impegno di Strina per dotare la comunità caprese di un luogo destinato all'educazione e all'accoglienza delle donne va collegato al clima isolano caratterizzato dalla rissosità tipica dei piccoli e deprivati centri del Mezzogiorno. Una realtà variegata e discontinua, nella quale la sostanziale tenuta sociale era affidata soprattutto alla rete devozionale aggregata attorno a istituzioni religiose e opere pie.

Capri non si sottraeva a questa cifra comune, anche se molto forte si erano dimostrati alcuni radicamenti civici della piccola comunità. Non è un caso, per esempio, che le aspre tensioni tra il vescovo Pellegrino e i rappresentanti dell'università si accendessero a seguito del tentativo del presule di acquisire il controllo della gestione patrimoniale del Monte di S. Giuseppe. L'istituzione assistenziale provvedeva al pagamento annuale di due maritaggi destinati alle fanciulle dell'isola, secondo una tradizione diffusa in tutti i centri urbani del Regno. Nella fattispecie, però, non si trattava di un'opera pia collegata alla cultura caritativa di matrice religiosa o alle reti di appartenenza professionale o di ceto, ma piuttosto di un meccanismo di sostegno alla crescita cittadina radicato nell'identità locale. Istituito nel parlamento del 3 giugno 1640 e finanziato dalla tassa sul grano, il sussidio all'ordinaria dinamica della comunità aveva dunque una chiara connotazione laica e civile 6 . Per questo, i rappresentanti del governo ricorsero al Collaterale per rivendicare l'autonoma gestione del Monte e del suo patrimonio, ricevendo il sostegno delle istituzioni della capitale e del viceré Medina de las Torres. Le oratorie trasmesse al Pellegrino dal 1642 riguardarono anche altri episodi della vita caprese e inasprirono quel confronto col presule che, protrattosi negli anni a venire, avrebbe più volte lasciato la diocesi priva del suo vescovo, allontanato e sostituito da vicari apostolici sorvegliati dall'arcivescovo di Amalfi.

In questo tessuto sociale deprivato, si aprivano, come si dirà più avanti, ampi spazi per le sollecitazioni carismatiche e per iniziative volte a costituire luoghi di edificazione spirituale e di radicamento comunitario. Una tale cifra, però, era molto lontana dal progetto di Marcello Strina, uomo di Chiesa e di rigida osservanza controriformata, al servizio della comunità parrocchiale anche per i bisogni non strettamente correlati all'impegno pastorale ${ }^{7}$.

Nel suo testamento, rogato il 10 agosto 1656 dal notaio Giovanni Battista Ignazio Fontanabona, egli nominava erede universale la sorella, ma stabiliva alcuni legati tra i quali quello a favore di

Galasso, A. Valerio (eds), Donne e religione a Napoli. Secoli XVI-XVIII, Milano 2001, pp. 139-158 e 210-237.

6 La documentazione istitutiva del Monte si trova in ASNa, Cappellano Maggiore 1182, fasc. 21. Un apologetico resoconto della vicenda in: G. Ruocco, Capri e Filippo IV di Spagna nel vicerealismo del Regno di Napoli attraverso $i$ superstiti monumenti storici di mons. Paolo Pellegrino alla seconda metà del secolo XVII, Napoli 1956. Per una trattazione aggiornata del tema: D. Casanova, Le porte del Paradiso. Le confraternite napoletane in età moderna, Napoli 2014; V. Fiorelli, Reti della dedizione e identità senza Stato, in R.M. Delli Quadri (ed), Storia e identità storica nello spažio euromediterraneo, Napoli, Guida 2015, pp. 51-64.

7 Le fonti, per esempio, registrano Strina tra coloro che si contrapposero all'infeudazione tentata da Antonio Barrile a favore della continuità demaniale e testimoniano l'impegno, prima di essere egli stesso contagiato, a favore della comunità durante l'epidemia di peste. La lite tra il duca di Marianella è l'università è in ASNa, Sezione Giustizia, Pandetta Nuova II, vol. II, 276-15. 
Prudenza. A lei, il sacerdote lasciava la sua casa perché ne facesse un monastero femminile, insieme alle rendite necessarie a realizzare il progetto, ma poneva delle rigide clausole perché la nipote potesse entrare in possesso del lascito. Le disposizioni testamentarie e il codicillo, successivo di soli quattro giorni, stabilivano infatti che il nucleo iniziale della nuova congregazione dovesse essere composto di almeno otto donne e che la nuova comunità doveva osservare la «regola di Santa Teresa», delineando ab initio un percorso dal profilo istituzionale di lungo periodo e non il semplice sostegno al progetto individuale di un membro della famiglia ${ }^{8}$. L'originaria affiliazione carmelitana, infatti, non costituiva tanto un obiettivo, quanto lo strumento necessario a radicare la nuova istituzione nell'alveo di un ordine regolare riformato il cui rigore appariva al testatore una garanzia per trasformare la comunità devota in un vero monastero di clausura.

Subentrato al parroco caprese nella direzione spirituale di Prudenza, don Ottavio avrebbe piuttosto privilegiato un percorso di legittimazione della personalità carismatica della donna, sigillo necessario alla figura della monaca fondatrice affidata alla tutela di un ordine religioso maschile, gli oratoriani, che avevano a Capri un radicamento né recente né estemporaneo. Le fonti testimoniano la presenza di una casa dei padri dell'Oratorio destinata a periodi di convalescenza o di allontanamento e i sedici anni di episcopato di Troiano Bozzuto, proveniente dalla casa napoletana, dovevano aver contribuito a rinsaldare quel legame ${ }^{9}$. Ma ancora più determinante sarebbe diventata la presenza oratoriana accanto alla monaca caprese, come testimoniano le cospicue donazioni di numerosi confratelli a favore del monastero del SS. Salvatore, o il suo ripetuto affidamento a confessori dell'Oratorio tra i quali un ruolo importante rivestirono Antonio Glielmo, Vincenzo Avinatri, Nicola Squillante e Tommaso Pagani, autori, questi ultimi, della sua settecentesca agiografia ufficiale ${ }^{10}$.

8 «Item lascia a suor Prudenza Pisa la sudetta sua casa, dove al presente abita esso testatore purché se ne facci Monastero o Casa di ritiramento di Donne sotto il titolo del SS.mo Salvatore e non effettuandosi frà termine d'anni diece dopo seguita sua morte detto Monastero o casa di ritiramento ut supra detta casa sia della sudetta sua erede ... quale Monastero abbia da essere fondato con tutti li requisiti necessari per il mantenimento di esso; quali Donne che si ritireranno in esso Monastero abbiano da osservare la regola di S. Teresa e siano di numero di almeno otto e così si debbia osservare e non altrimenti. Di più lascia al sudetto Monastero effettuantosi ut supra perfezionata sarà docati mille una con l'interessi, ed ogn'altro che ad esso testatore li spettano come erede della quondam Beatrice Cursia ... Et più lascia al detto monastero perfezionato ... dopo la morte della sudetta Anna sua sorella il Truglio ch'esso testatore possiede sopra la Marina grande di detta isola di Capri». La trascrizione del testamento e del codicillo è inclusa nel manoscritto Memorie istoriche dell'origine e progressi del venerabile monastero del SS. mo Salvatore della città di Capri fondato dalla Ven. Madre Serafina di Dio. Scritte nell'anno 1750, in BCIC, Archivio 10.

9 Esemplare, per esempio, il racconto settecentesco dell'episodio di possessione di un novizio nel quale fu coinvolto Nicola Squillante, confessore oratoriano di Madre Serafina. Delle tre versioni della cronaca è stata recentemente pubblicata quella intitolata Caso successo nella Casa de' P.P. Gerolimini in Napoli à IV del mese di Maggio 1696: A. Fienga (ed), Satana in convento. Curiosa narrazione di diabolici eventi occorsi a Napoli nella casa dei PP. Oratoriani l'anno MDCXCVI, Napoli 1992.

10 Squillante era stato maestro dei novizi, cerimoniere, corettore a mensa e prefetto della congregazione dei dottori nell'Oratorio di Napoli. La vita di Prudenza, interrotta per la sua morte nel 1719, fu terminata dal confratello Pagani. Il titolo completo dell'opera è: N. Squillante, T. Pagani, Vita della venerabile madre suor Serafina di Dio fondatrice di sette monasteri dell'ordine carmelitano. Incominciata a descriversi dal p. Nicolò Sguillante della congregazione dell'Oratorio di Napoli ma per la morte di 
Le fasi organizzative iniziali per la nuova comunità si svolsero tra Napoli e Capri e furono funestate da resistenze crescenti negli ambienti isolani destinati ad accoglierla, superate grazie alla collaborazione di Pisa con l'arcidiocesi di Amalfi, sostenitrice attiva del progetto della prima fondazione, ma anche della successiva diffusione delle congregazioni istituite da Madre Serafina lungo la costa prospiciente l’isola di Capri.

La lettura delle fonti coeve traccia una situazione in netta controtendenza rispetto al clima di acceso devozionismo di metà Seicento, quando la diffusione delle fabbriche conventuali trasformava il volto stesso di città e piccoli centri in teatri della Chiesa trionfante. La crescita del valore identitario attribuito alla presenza religiosa femminile nei contesti urbani e nella cultura sociale postridentina era accentuata dal bisogno di rassicurazioni collettive provocato dal flagello della peste che aveva favorito la disseminazione di chiese, monasteri, ma anche semplici edicole votive, tutte indistintamente percepite come segni tangibili del trascendente nel tessuto urbano ${ }^{11}$. Capri, invece, stentava a riconoscere il ritiro affidato da Strina alla nipote come un elemento organico al proprio tessuto sociale. Questo in parte perché i riferimenti istituzionali di Prudenza erano oramai esterni al perimetro isolano, ma anche perché, nonostante l'ideale affiliazione all'ordine carmelitano, la nuova istituzione sottoposta al controllo diocesano veniva a rinsaldare e potenziare la posizione del vescovo Pellegrino in guerra aperta con l'università.

Mentre don Ottavio s'impegnava nella capitale per costituire una rete di piccoli finanziatori in grado di aggirare le resistenze della comunità caprese, suor Tenza, come usava affettuosamente chiamarla il prelato, cercava adepte per il primo ingresso nel ritiro penalizzato dalla scarsa accoglienza da parte degli ambienti più influenti dell'asfittico tessuto locale ${ }^{12}$.

questo proseguita e data alla luce dal p. Tomaso Pagani della medesima congregazione, Napoli 1723. La prima fonte per la storia degli oratoriani a Napoli è G. Marciano, Memorie Historiche della Congregazione dell'Oratorio, Napoli 1693-1702, ma si veda anche A. Cistellini, San Filippo Neri. L’Oratorio e la congregażione oratoriana. Storia e spiritualità, Brescia 1989.

11 Troppo vasta la bibliografia relativa a questi temi per darne conto in questa sede. Mi sia però consentito rinviare a V. Fiorelli, Superbi palaggi. Il contributo dei governi diocesani alla costrųione delle identità cittadine nella Campania moderna, in A. Musi (ed) Le dimore signorili nel Regno di Napoli: l'età spagnola, Fisciano 2014, pp. 229-240.

12 Le difficoltà iniziali erano chiare per Pisa: "tanto in Napoli, quanto nel Isola istessa molti e biasimavano e contraddicevano a tal opera, dicendo, et anche con beffe alcuni, ch'era una pazzia, un'imprudenza, che mai haverebbe potuto riuscire ne mantenersi per non esserci buon fondamento di facoltà ne di gente che vi sarebbero andate non trovandoci nel'Isola quelle comodità ne temporali ne spirituali di chi potesse aiutarlo». Pisa, Libro del Monasterio, f. 6. Secondo la testimonianza di suor Ammirabile della Solitudine, al secolo Lucia Vona, e suor Arcangela Fortunata, la nipote di Prudenza Teresa Martorelli, autrici dei due volumi della biografia della Madre voluta dal confessore Avinatri, questa aveva adottato una strategia di lungo periodo di affiliazione al monastero: «alcune figliole che nascevano in quel tempo et allevarle al servitio di Dio per dovere poi servire per il monastero. E per questo disegno à molte di queste fece mettere il nome Teresa». Questo manoscritto proveniente dalla chiesa di S. Stefano è in deposito temporaneo presso il CADIC ed è indicato Memorie, t. I, f. 11. Sul tema: V. Fiorelli, Da Capri alla terraferma. L'esperienza di un «centro periferico» nei monasteri fondati da madre Serafina di Dio in E. Novi Chavarria (ed), La città e il monastero. Comunità femminili cittadine nel Mezzogiorno moderno, Napoli 2005, pp. 281-300. 
Se il coinvolgimento attivo del canonico napoletano nel percorso iniziale del conservatorio aveva contribuito alla percezione di un corpo estraneo da parte dei capresi, la scelta di Strina di vincolare l'istituendo ritiro alla spiritualità teresiana e a un ordine in piena espansione nella capitale costituiva un ulteriore elemento di sradicamento al quale non era probabilmente estranea la connotazione filospagnola della rete carmelitana partenopea. Non esistono evidenze documentarie in tal senso, ma si può verosimilmente ipotizzare che la disposizione del parroco caprese fosse in parte motivata da un modello di congregazione femminile che, consentendo un impegno di apostolato attivo, aveva offerto esempi evidenti di resistenza allo sforzo di omologazione e di disciplinamento della Chiesa controriformata, ma anche da una scelta di lealismo utile a garantire un più lineare percorso di realizzazione nel conflittuale contesto isolano.

É noto il ruolo svolto dal viceré Juan Alonso Pimentel de Herrera, in viaggio verso la sua nuova sede, nel trasferimento da Genova del drappello carmelitano che avrebbe fondato il monastero di S. Giuseppe delle Scalze a Pontecorvo. É altrettanto chiaro, poi, l'intento politico che aveva motivato il sostegno del conte di Benavente alla diffusione del messaggio teresiano nel Regno ${ }^{13}$. Si trattava, infatti, di un'iniziativa coerente con quella più generale tendenza a rafforzare l'omogeneità culturale all'interno della monarchia composita degli Asburgo di Spagna facendo leva sulla forza identitaria della religione nelle comunità sociali di entrambe le penisole. Un'osmosi continua di modelli sociali e di schemi politici sulla quale s'interroga il più aggiornato dibattito storiografico, con il merito di aver riportato l'analisi degli intensi rapporti tra le due penisole del Mediterraneo nella più ampia prospettiva transnazionale dell'histoire croisée. Definitivamente archiviata la stagione dominata dall'antispagnolismo e dalla lettura di un secolo XVII segnato dalla decadenza e dalla corruzione provocate, nello spazio italiano, dal predominio iberico e dall'influenza della Chiesa, dunque, le ricerche sulla storia religiosa del Mezzogiorno hanno ricominciato a interrogarsi sulla possibilità di tracciare i confini tra assimilazione e autonomia culturale nei rapporti tra le due capitali, verificando la continuità e la riconoscibilità di ciascuna identità culturale garantita attraverso una contaminazione che non si è mai trasformata in fusione, nonostante la subalternità politica di Napoli a Madrid ${ }^{14}$.

Questa prospettiva è stata ampiamente sottolineata nelle ricerche sull'editoria spagnola a Napoli e sul ruolo non secondario ricoperto dalle edizioni partenopee delle opere di Teresa d'Avila promosse dai carmelitani. La prima edizione integrale degli scritti della santa realizzata, in castigliano, fuori dalla

$13 \mathrm{Al}$ primo insediamento carmelitano riformato, realizzato nel 1606 da cinque religiose riformate accompagnate da tre sacerdoti e un chierico, si sarebbero affiancati S. Maria Antesaecula nel 1628 e, quattro anni più tardi, Santa Maria del SS. Sacramento. Per un inventario delle fonti d'archivio e i riferimenti bibliografici si veda ad vocem A. Valerio (ed.), I luoghi della memoria, 2vv., Napoli 2006-2007, v. II, Istituti religiosi a Napoli dal 1600 al 1861, 2007. Sul ruolo dei viceré: D.A. Parrino, Teatro eroico e politico de' governi de' viceré del regno di Napoli, 3 vv., Napoli 1692-1694, v. I, 1692, p. 211.

14 Mi limito qui a rinviare alle considerazioni espresse da Giuseppe Galasso in Aspetti dei rapporti tra Italia e Spagna nei secoli XVI e XVII, «L’Acropoli», 5/2013, pp. 446-467 e agli studi raccolti in A. Musi (ed), Alle origini di una nazione. Antispagnolismo e identità italiana, Milano 2003. Cfr. V. Fiorelli, Transiz̨ioni a Mezzogiorno. Prospettive e tradiz̧ioni di ricerca sulla cultura religiosa e la pratica devota tra storia e storiografia, in «Nuova rivista storica», 3/2014, pp.1157-1176. 
Spagna, infatti, ha contribuito in modo determinante alla diffusione in Italia del suo carisma spirituale, ma anche al rafforzamento della dimensione identitaria dell'ordine nell'alveo comune della politica religiosa e devozionale perseguita dalla Corona ${ }^{15}$.

Questo taglio storiografico è ampiamente condiviso dai numerosi studi che hanno analizzato la valenza politica e culturale del linguaggio della ritualità utilizzato come chiave di lettura dei rapporti tra Napoli e la Spagna ${ }^{16}$. Per limitarci a un accenno collegato alla presenza carmelitana, di grande suggestione ci sembra la cronaca del sacerdote Andrea Rubino nelle pagine dedicate alla descrizione delle sontuose cerimonie pubbliche organizzate nel 1664 in occasione dell'elevazione di santa Teresa tra i patroni nella Napoli governata dal viceré Gaspar de Bracamonte y Guzmán conte di Peñaranda ${ }^{17}$.

Se l'affiliazione al magistero spirituale della santa di Avila era parte del progetto originario del monastero caprese, dunque, le norme che regolavano la vita della prima comunità di devote erano state stabilite da Ottavio Pisa nel corso dei colloqui con la sua figlia spirituale, quando ancora si attendeva l'autorizzazione del vicario apostolico per il progetto della casa di ritiro a modello di monastero ${ }^{18}$. Queste consuetudini, che le monache rispettavano fin dal 1661, furono messe per iscritto solo molti anni dopo, quando presero la forma definitiva di una regola carmelitana mitigata e iniziarono, nel 1676, l'iter per l'approvazione pontificia, insieme alla richiesta di clausura ${ }^{19}$.

Da un punto di vista della percezione dei contemporanei, non vi erano incertezze sulla matrice teresiana dei conventi fondati da madre Serafina. Nel 1667 il monastero del Salvatore fu registrato

15 Sulle edizioni delle opere di santa Teresa realizzate a Napoli si veda E. Sánchez García, Observaciones sobre el linaje de Santa Teresa: la edición partenopeas de las Obras y otras iniciativas, in P.M. Piñero Ramírez (ed), Dejar ablar a los textos. Homenaje a Francisco Márquez Villanueva, 2 vv., Sevilla 2005, v. I, pp. 473-496. Cfr. Ead., Producción impresa hispánica en el Reino de Nápoles (1503-1707), in P. Botta (ed), Rumbos del hispanismo en el umbral del Cincuenteneraio de la AIH, 3vv., Roma 2013, v. III, pp. 338-346.

$16 \mathrm{Si}$ veda, per esempio, la questione dello sviluppo politico della devozione dell'Immacolata per il quale mi limito a rinviare a A. Prosperi, L'Immacolata a Siviglia e la fondazione sacra della monarchia spagnola, in I. Fazio, D. Lombardi (eds), Generazioni. Legami di parentela tra passato e presente, Roma 2006, pp. 125-162; P. Broggio, Immacolata Concezione, in «Bruniana e Campanelliana», 1/2011, pp. 233-243. Vastissima è la bibliografia sul linguaggio rituale che ha registrato di recente una grande vitalità degli studi. Mi limito pertanto a ricordare G. Galasso, J.V. Quirante, J.L. Colomer (eds), Fiesta y ceremonia en la corte virreinal de Napoles (siglos XVI y XVII), Madrid 2013; G. Guarino, Public Ritualsand festivals in Naples, 1503-1799, in T. Astarita (ed), A Companion to Early Modern Naples, Leiden-Boston 2013, pp. 257-280.

17 Fonte insostituibile per la storia napoletana, il manoscritto Notitia di quanto è occorso in Napoli dal 1448 per tutto l'anno 1657 è conservato in SNSP, mss. XXIII.D.14- 17. Su Rubino si veda almeno I. Mauro, Crónica festiva de la Nápoles virreinal. La Notitia de Andrea Rubino (164 8-1669), in « Cuadernos de Historia Moderna», 34/2009, pp. 67-93.

18 «Si ritiravano insieme ambi D. Ottavio e lei, e con la penna alle mani si scrivea quello che di comune consenso meglio parea: e molte volte nel altre sessioni ruminando quello ch'haveano scritto alle prime o si moderava o si agiungeva alcuna cosa, e così fu seguitato massime per tutte le regole communi e parte delle Costituzioni». Pisa, Libro del Monasterio, f. 11.

19 Mai arrivate alle stampe, esse circolavano in forma manoscritta nei conventi delle teresiane in versioni omogenee che richiamavano nella forma e nella sostanza la filiazione dalle Regole del Carmelo. Una copia della Regola di S. Teresa proveniente dal monastero di Capri è rilegata nel volume Memorie, t. II, ff. 382-439v.

SCRIPTA, Revista internacional de literatura i cultura medieval i moderna, núm. 6 / desembre 2015 / pp. 155 - 165 ISSN: 2340-4841 · doi:10.7203/SCRIPTA.6.7828 
per la prima volta in una relazione ad limina con la dizione «Conservatorium Monialium [...] sub habitu et Regula S. Theresiae» e l'abate Pacichelli indicava, nella sua descrizione di Capri, la presenza sull'isola di un «conservatorio per cinquanta religiose carmelitane» secondo una dizione che si ritrova nella letteratura dedicata alle descrizioni della penisola sorrentina, fino al riferimento di Filangieri al monastero di Nocera ${ }^{20}$.

Oltre alle sue fondazioni, anche la parabola carismatica di Serafina di Dio risentì profondamente della spiritualità di santa Teresa. Del modello della santa spagnola, infatti, la madre caprese aveva certamente assimilato la tendenza a rafforzare i legami devozionali con le consorelle e con i devoti attraverso un costante scambio epistolare e una copiosa scrittura spirituale. Quando, nel 1745, il cardinale Carlo de Marinis stese il Decretum super revisione et approbatione opuscolorum et aliorum scriptorum servae Dei editum die 15 Maij 1745, nell'elenco degli scritti superstiti della Madre raccolti tra Napoli e Capri e approvati con decreto della Sacra Congregazione dei Riti del 15 maggio dello stesso anno risultavano 2167 lettere, diversi trattatelli spirituali e scritti di pratiche devozionali. Una caratteristica della spiritualità della monaca caprese che riproduceva la capacità teresiana di trasformare il carisma della parola scritta nella forza e nell'autorevolezza necessaria a sostenere una vasta rete di iniziative di fondazione $e^{21}$.

Dopo essere riuscita a costruire, nel piccolo centro dell'isola di Capri, un imponente convento dotato di tutte le caratteristiche necessarie per diventare un monastero di clausura di prima importanza, riferimento per gli ambienti professionali e mercantili della capitale, Serafina fu chiamata a porre il suggello spirituale e il contributo organizzativo ad altre sei congregazioni

Tre di essi nascevano sotto l'egida dei vertici diocesani e il sugello carismatico della Madre caprese offriva loro un potenziamento devozionale per iniziative di chiara matrice ecclesiastica. L'apertura, nel 1673, del conservatorio di Santa Teresa a Massa Lubrense, per esempio, aveva ripercorso la strada del convento caprese. Un primo gruppo di devote raccolte nella casa di famiglia dei Pisa fu trasformato in congregazione dalla sinergia tra don Ottavio e il vescovo Francesco Maria Neri, responsabile della diocesi di Capri durante uno dei periodi di allontanamento di Pellegrino, grazie alla imposizione di una regola di matrice teresiana ${ }^{22}$. Un percorso simile avrebbe seguito il convento

20 La visitanon fu effettuata dal Pellegrino ma dal canonico Antonio Varano. La citazione per esteso è: «Conservatorium Monialium sub titulo SSmi Salvatoris sub habitu et Regula S. Theresiae quod fuit fundatum de elemosinis, in absentia nostra, licet unum consensu vicarijs Apostolici tunc temporis iurisdictionem habentis», f. 144r. La riproduzione dell'originale Relatio ad limina del 1667 è consultabile in CADC.

$21 \mathrm{Si} \mathrm{veda} \mathrm{a} \mathrm{questo} \mathrm{proposito} \mathrm{l'apporto} \mathrm{di} \mathrm{alcuni} \mathrm{saggi} \mathrm{pubblicati} \mathrm{in} \mathrm{F.J.} \mathrm{Sancho} \mathrm{Fermín,} \mathrm{R.H.} \mathrm{Cuartas} \mathrm{Londoño} \mathrm{(eds),}$ El Libro de las Fundaciones de Santa Teresa de Jesús,), Editorial Monte Carmelo, Universidad de la Mística CITeS 2013. La trascrizione del decreto del 1745 è in BCIC, Archivio Carelli 294, II D, f. 77-80.

22 La regola seguiva il modello di quella in uso nel monastero del Salvatore e fu rogata dal notaio Giovanni Persico il 16 novembre 1673: Costituzioni e Regole del Monastero nuovamente eretto nella città di Massa Lubrense sotto la regola della Madre S. Teresa secondo la modificazione de' Sommi Pontefici Innocenzo IV, Eugenio IV e Sisto IV dall'antica della B. Vergine di Monte Carmelo. Per la storia delle singole fondazioni si veda Fiorelli, Una esperienza religiosa periferica cit., pp. 95-124. 
della SS. Trinità a Vico Equense, dove fu utilizzato un lascito testamentario della fine del secolo XVI. Ma ancora più netta appare la connotazione diocesana dell'intervento del 1685 a Torre del Greco dove, per volontà di Innico Caracciolo, l'arcivescovo di Napoli impegnato a riorganizzare il laicato devoto femminile dell'intera provincia, Serafina fu chiamata a trasformare in una comunità regolata la congregazione di bizzoche che, per motivi sostanzialmente economici, non si era riusciti ad agganciare alla regola domenicana del monastero napoletano di Santa Caterina da Siena ${ }^{23}$.

Una riconversione ecclesiastica di iniziative laiche radicate nel tessuto sociale locale, finanziate da singoli devoti o da reti urbane ben riconoscibili, caratterizzava invece i percorsi di fondazione di Santa Maria della Purità a Nocera de' Pagani nel 1680, quello di S. Michele Arcangelo ad Anacapri tre anni dopo e quello di S. Giuseppe a Fisciano nel 1691.

Se ognuna delle nuove realtà si inseriva in uno specifico contesto storico e sociale, però, per ciascuna madre Serafina cercò di seguire procedure omogenee per imprimere un profilo comune ai conservatori che si riconoscevano nel suo magistero spirituale. Come, nel 1606, le carmelitane di Genova erano venute nel Regno a garanzia del trasferimento di un'autentica matrice teresiana, così Serafina si spostava con alcune fedeli religiose che poi lasciava nella nuova sede a sorvegliare il percorso di consolidamento, ma soprattutto la continuità del modello del SS. Salvatore. Un meccanismo che consentiva alla fondatrice di mantenere un controllo diretto sui nuovi conservatori e di governarne, da Capri, gestione e organizzazione, ma soprattutto le garantiva di istituire reti devozionali saldamente dipendenti dal suo carisma secondo un meccanismo di affiliazione spirituale nel quale resta assai difficile determinare i confini tra una vita modellizzata dalla santità e il progetto di santificazione della propria vita.

Non è dunque né strano né inaspettato che, nel pieno della sua attività di promozione istituzionale, la religiosa attirasse l'attenzione della Congregazione Romana che la sottopose a una lunga indagine dalla quale Serafina non sarebbe mai veramente uscita. Durante gli ultimi anni della sua vita, infatti, fu travolta da una denuncia presentata al Sant'Uffizio di Napoli contro Francesco Cesari.

L'inchiesta che la vide «successivamente carcerata indiciata di duoi deletti, d'uno cioè d'affettata santità fondato in visioni rivelazioni e predizioni [...], et l'altro di proposizioni contenute in alcuni quinternetti scritti di sua mano» durò dal 1685 al 169124. Dopo tredici anni di servizio presso l'Oratorio di Napoli, Cesari era diventato seguace di Miguel de Molinos e si era ritirato a Capri nel dove aveva iniziato «ad istruirvi alcune zitelle nell'Oratorio di Quiete». Serafina era venuta in contatto con il religioso attraverso la sua consuetudine con gli oratoriani e l'impronta carismatica

23 Si veda a questo proposito: G. Boccadamo, Monache di casa e monache di conservatorio, in Donne e religione a Napoli cit., pp. 159-191.

$24 \mathrm{Il}$ fascicolo del processo è conservato in ACDF, S. O., St. st. C 2-e, f. 9. Su questo, oltre al più volte citato volume Una esperienza religiosa periferica, si veda V. Fiorelli I sentieri dell'inquisitore. Sant'Uffizio, periferie ecclesiastiche e disciplinamento devozionale (1615-1678), Napoli 2009; Ead., ad vocem in A. Prosperi, J. Tedeschi (dir.) Dižionario storico dell'Inquisiz̨ione, 4 vv., Pisa 2010, v. III, pp. 1412-1413. 
della sua proiezione pubblica la fece naturalmente sospettare di avere avuto una parte rilevante nel progetto di raccogliere, attorno al fuoriuscito dell'Oratorio, una comunità femminile di preghiera, coinvolgendola nella grave accusa di avere simpatie quietiste, con grave danno del suo ruolo di guida spirituale ${ }^{25}$. L'indagine fu inizialmente seguita da David Scribani, ministro del Sant'Uffizio a Napoli dal 1684 al 1686. La delicatezza della situazione che, ancora una volta, vedeva pesantemente coinvolti i seguaci di s. Filippo, rese opportuno il suo trasferimento a Roma dove il destino della madre caprese fu presto separato da quello del quietista dichiarato.

Durante le indagini, furono soprattutto gli scritti di madre Serafina a essere sottoposti a una puntuale e rigorosa valutazione. L'accusa di quietismo cadde quasi subito in quanto, senza incertezze, la carismatica aveva sempre rivendicato la funzione del suo magistero nell'alveo della Chiesa diocesana, dissociandosi tanto dalla linea spirituale di Molinos, quanto dalla vita della congrega laicale di donne organizzata al di fuori dell'istituzione ecclesiastica. Per ridare legittimità al suo magistero, gli agiografi, le consorelle che preparavano un canovaccio per il processo di canonizzazione, ma anche la sua stessa narrazione del percorso devoto predisposto per rafforzare la statura istituzionale delle «sue» teresiane, cercarono soprattutto di descrivere un meccanismo di scrittura automatica, inconsapevole e ispirata da Dio, radicato nella tradizione della santa illetterata, necessario per stemperare la forza di una direzione spirituale femminile che, sul modello della santa di Avila, aveva voluto lasciare il segno nella pagina scritta.

Ciò nonostante, dal 1687, le fu impedito di lasciare il convento e i suoi atteggiamenti continuarono a destare viva preoccupazione negli inquisitori. La vicenda penale si concluse con la censura formale e la distruzione di quasi tutta la sua produzione letteraria, nonché con la condanna a un'abiura de levi che si accompagnò al divieto di esercitare ruoli direttivi nella comunità dove Serafina continuò a vivere, controllata dalle consorelle e da un confessore indicato dall'arcivescovo di Napoli, fino alla sua morte, avvenuta nel 1699 .

Una vicenda personale, quella della monaca fondatrice di Capri, che si è chiusa nell'oblio imposto dalle ragioni del disciplinamento, ma soprattutto per il ritardo del modello carismatico individuale nella temperie di fine secolo che apriva a una Chiesa nella quale l'affiliazione alle reti regolari offriva alle istituzioni locali la vera prospettiva di legittimazione istituzionale.

25 Per un rinvio bibliografico aggiornato su Molinos e il quietismo si veda ad vocem in Dižionario storico dell'Inquisiz̨ione cit. 